\title{
Flexible Spandrels of the Global Plant Virome: Proteomic-Wide Evolutionary Patterns of Structural Intrinsic Protein Disorder Elucidate Modulation at the Functional Virus-Host Interplay
}

\author{
Tahzima $^{1}$, R., Haegeman ${ }^{2}$, A., Massart, S $^{1}$, Hébrard, E. ${ }^{3}$ \\ ${ }^{1}$ University of Liège (ULg), Department of Integrated and Urban Phytopathology, 5030 Gembloux, \\ Belgium. rachid.tahzima@uliege.be
}

${ }^{2}$ Flanders Research Institute for Agriculture, Fisheries and Food (ILVO), Plant Sciences Unit, 9820 Merelbeke, Belgium.

${ }^{3}$ PHIM, Plant Health Institute, IRD, Cirad, Université de Montpellier, INRAE, Institut Agro, 911 avenue Agropolis, BP 6450134934 Montpellier, France.

Intrinsically disordered proteins and regions (IDPs/IDRs) make up a large part of viral proteomes, but their real prevalence across the global plant virome are still poorly understood, partly because of their massive diversity. Here we propose an evolutionary quantitative proteomic approach to foray into genomic signatures that are preserved in the amino acid sequences of orthologous IDRs. Markedly, we found that relatively abundant IDP varies substantially between viral species and within plant virus families, including according to genome size, partition or replication strategies. We also demonstrate that most encoded proteomic modules of the plant virome contain multiple disordered features that are phylogenomically preserved, and can be correlated to genomic, bio-physical and evolutionary strategies. Furthermore, our focused interactome-wide analysis highlights lines of evidence indicating that various IDPs with similar evolutionary signatures modulate viral multifunctionality. Moreover, estimated fractions of IDR in the vicinity of pivotal evolutionary structural signatures embedded in interaction modules are strongly enriched with affinity binding functional annotations and relate to vector-borne virus transmission modes. Importantly, Molecular Recognition Features (MoRFs) are abundantly widespread in IDRs of viral modules and their binding partners. Finally, we propose a coarse-grained conceptual framework in which evolutionary proteome-wide IDP/IDRs patterns can be, rather, reliably exploited to elucidate their foundational fine-tuning role in plant virus transmission mechanisms. While opening unexplored avenues for consistently predicting virus-host functions for many new or uncharacterized plant viruses based on their proteomic repertoire, other considerations advocating further structural IDP research in Plant Virology are thoroughly discussed in light of viral evolution. 

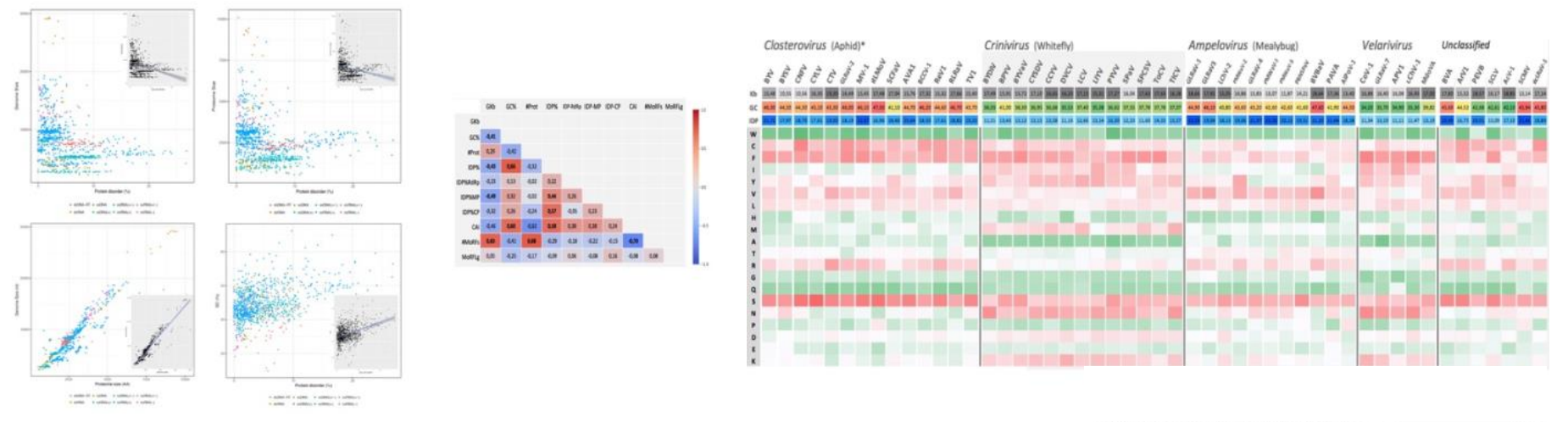

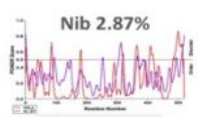

Andust

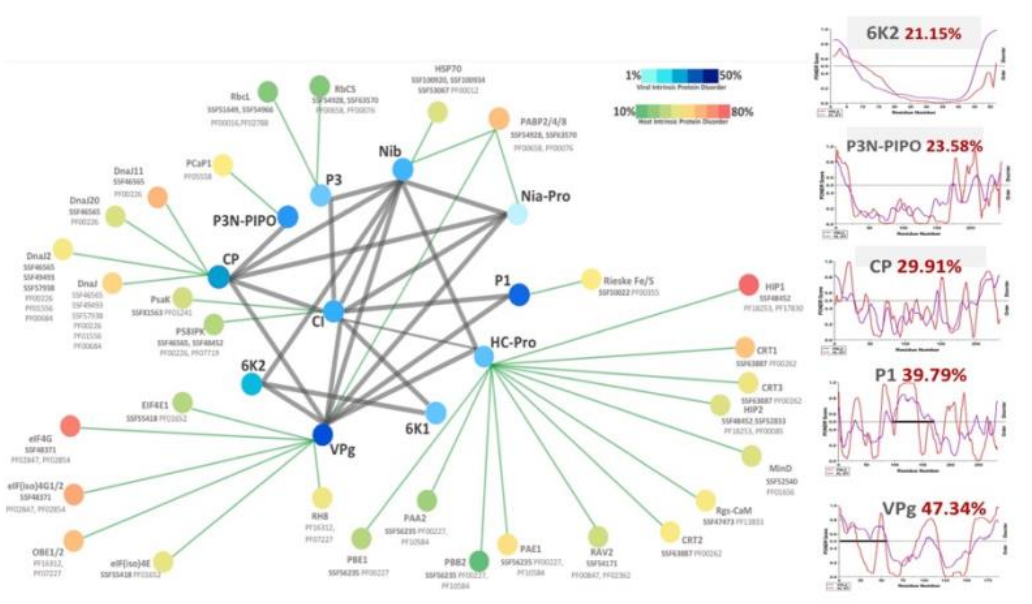

(1)

Fi: He-Pro 12.28\%

Cl 15.77\%

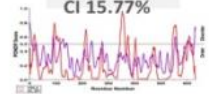

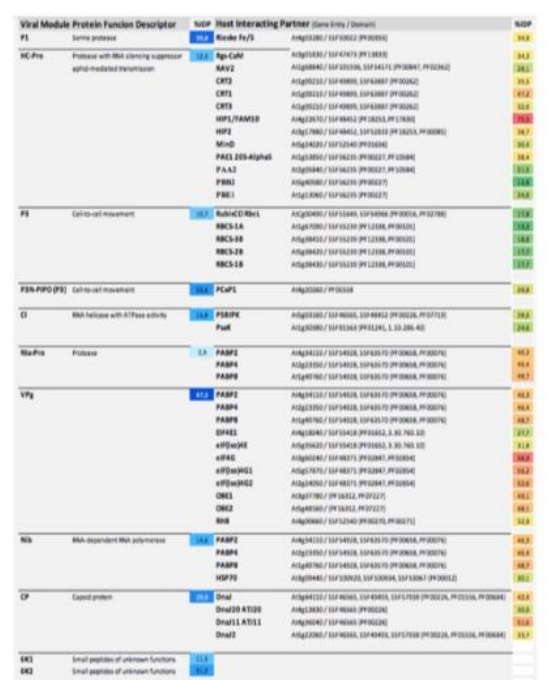

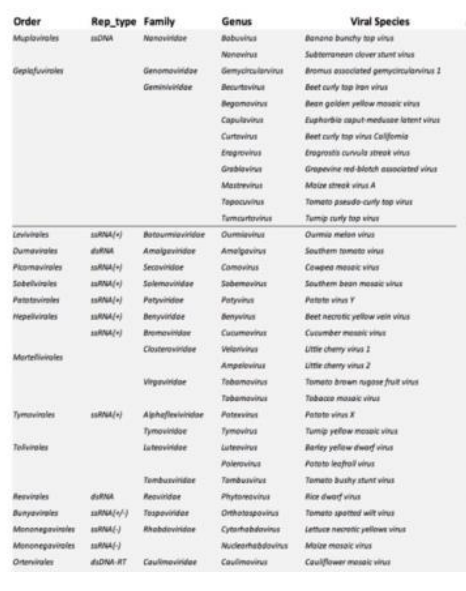
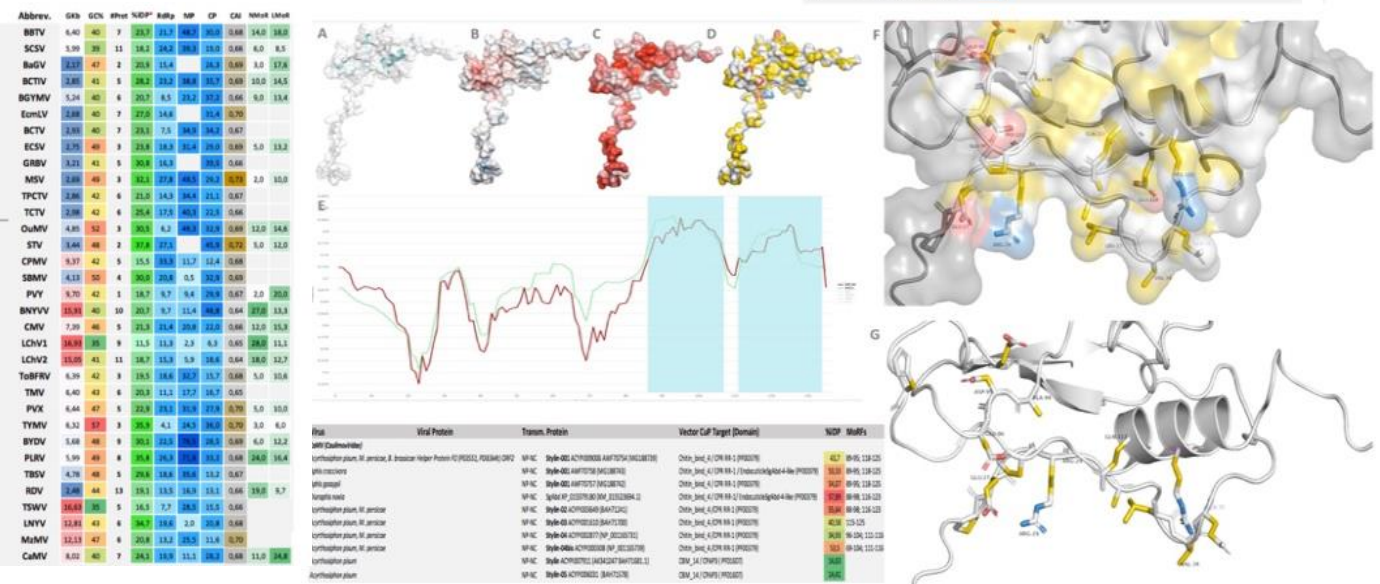

BYOV-CPm P09516
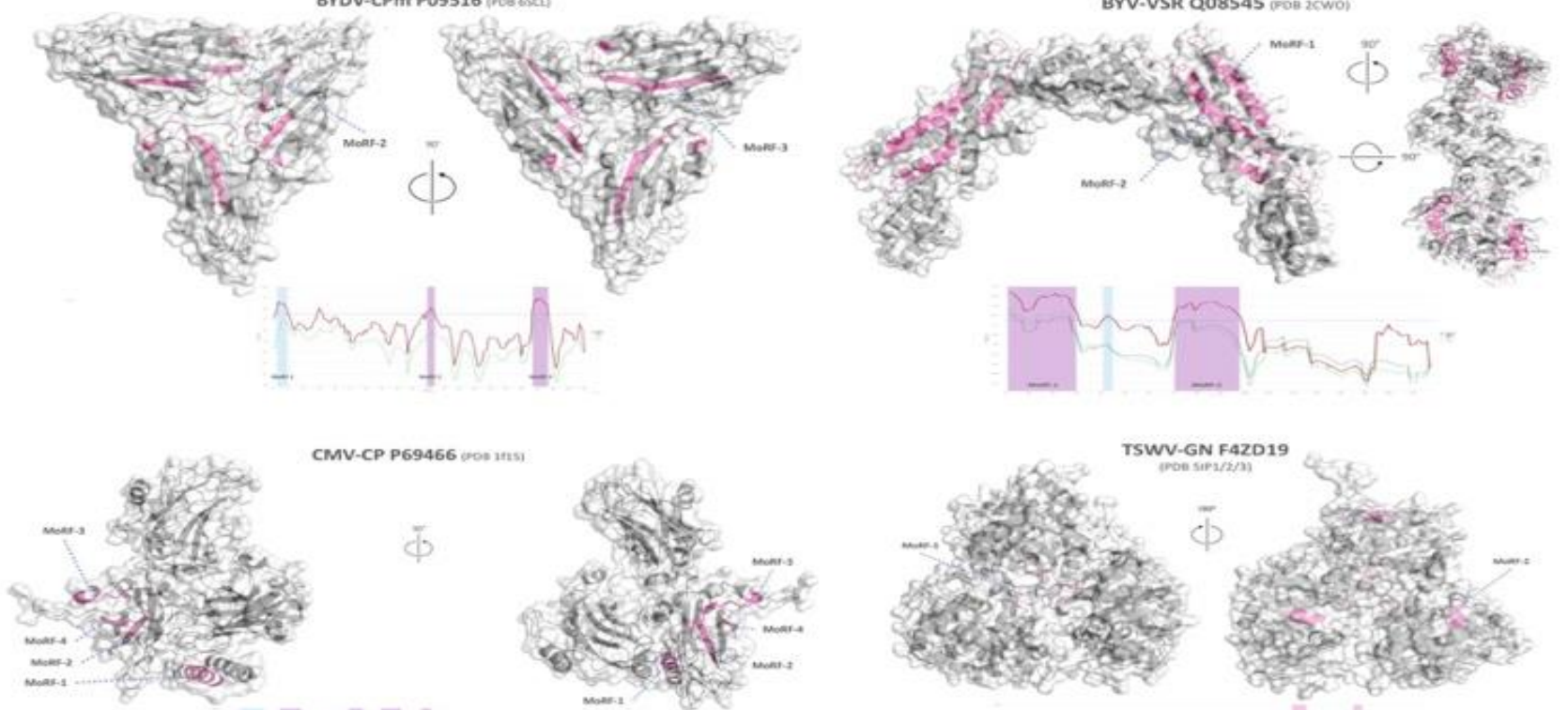

CMV-CP P69466 pos uns

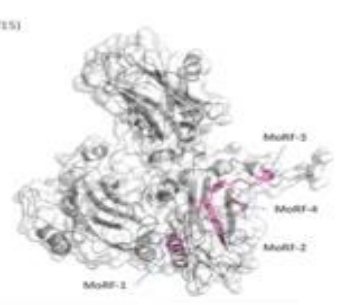

$$
\text { whtinma }
$$
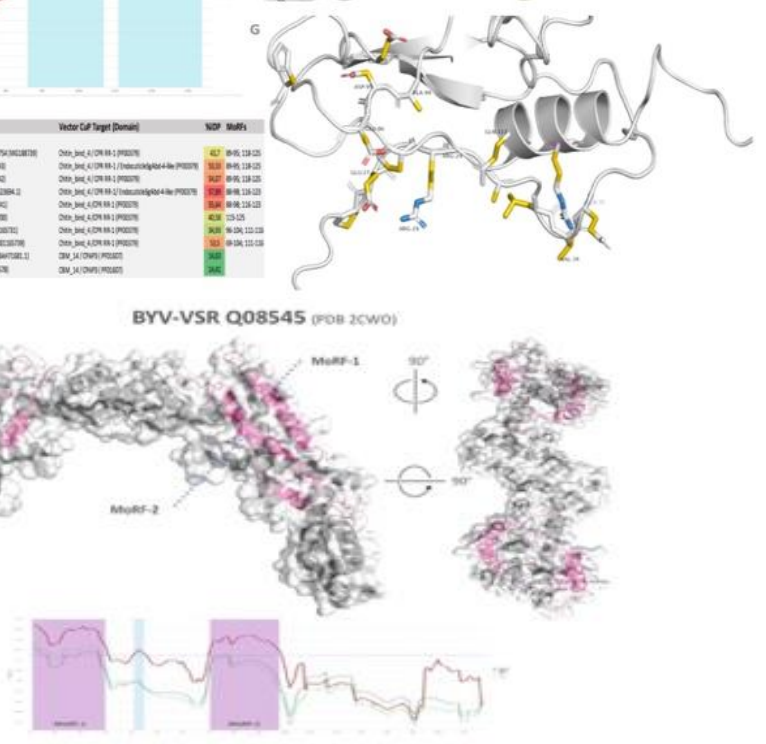

TSWV-GN F4ZD19

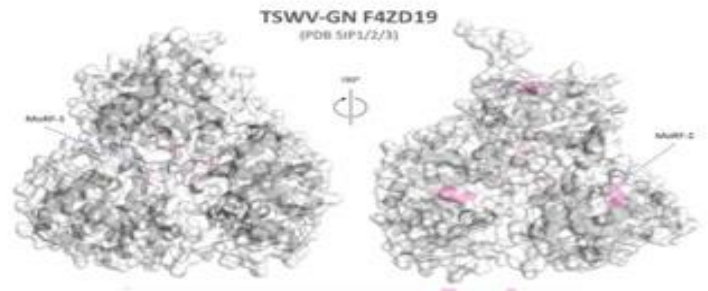

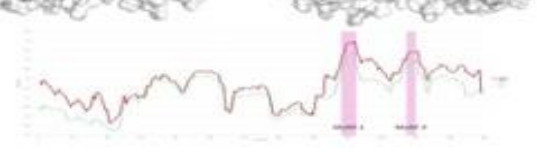

